\title{
Modeling of flow in microchannel with bubbles layer on surface
}

\author{
Dmitriy Gluzdov ${ }^{1, *}$ \\ ${ }^{1}$ Kutateladze Institute of Thermophysics, 630090 Novosibirsk, Russia
}

\begin{abstract}
Results of 2D numerical solution of liquid flow in microchannel with bubbles layers on surface are presented. Bubbles layers are modeled by setting of bubble size and Navier slip condition. Calculations have been done using OpenFoam PISO method. The results of modeling compared with analytical solution.
\end{abstract}

\section{Introduction}

Microtechnologies play important role in the modern world. Power of the integrate circuits grows every year, and its cooling becomes more difficult. Traditional cooling systems will be not able to effectively cool modern microchips in the near future. The demand of the liquid cooling systems grows, which can be successfully integrated into microchips using microchannels. Microchannels are an inherent part of liquid cooling systems as well as one of main obstacles on the way to increase their effectiveness. The problem of decreasing hydraulic resistance in the microchannel flows is actual issue nowadays.

The one of possible solutions reported in $[1,2,3]$. Reducing of hydraulic resistance will occur if the boundary surface has a grooves surface with gas, which allows liquid to slip. However, creating such structure is costly, and it leads to problem with maintaining gas in grooves. Moreover, the question of stability and rupture of liquid films on such substrates arises $[4,5,6]$.

Another possible solution to drug reduction is use of hydrophobic coatings for microchannel surfaces [7], where gas bubbles are collected. This gas bubbles have a height of several microns. The hydrophobic surface can significantly decrease adhesion; on the other hand, gas bubbles can reduce the volume for liquid flow, which can reduce a flow rate. So, the investigation to clarify the efficiency of using hydrophobic coatings is required.

The liquid flow in microchannel with Navier slip condition [8] and convex portions on hydrophobic parts modeling the configuration presented in [7] are simulated in 2D NavierStokes formulation. Calculations have been done using OpenFoam PISO method. The results of modeling compared with analytical solution.

*Corresponding author: gluzdov1@yandex.ru 


\section{Theory and the numerical solution}

Two-dimensional problem is considered. The scheme for calculation of microchannel flow is presented in Fig. 1. Hatching shows the hydrophobic parts.

Calculations made in OpenFoam. OpenFoam is an open integrated platform for numerical solutions. Method PISO (Pressure Implicit with Splitting of Operator) is chosen for calculations of full Navier-Stokes equations with Navier slip condition and convex portions on hydrophobic parts. The analytical solution of Poiseuille flow with the same boundary condition is used as a test of this method. The time of establishing steady state flow in this test is used. Error of this method does not exceed of $1 \%$.

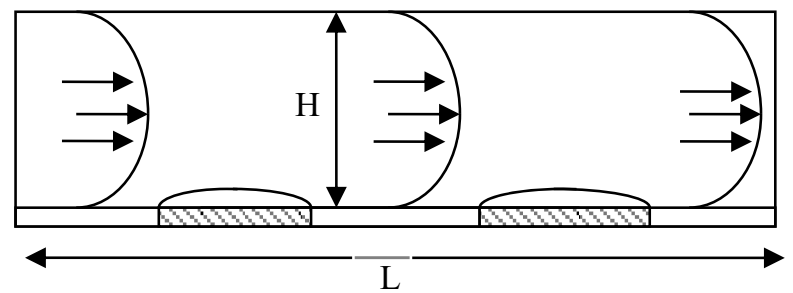

Fig. 1. Two-dimensional scheme for calculation of the flow in microchannel.

\section{Results}

Calculations are made for microchannel height $\mathrm{H}=0.5 \mathrm{~mm}$ and length $\mathrm{L}=100 \mathrm{~mm}$. Liquid is water with temperature of $293 \mathrm{~K}$. The bubbles are approximated by arcs with height less than $0.05 \mathrm{~mm}$. It was shown in [7] that the height of bubbles should be less than $30 \%$ of height channel for drug reduction.

Calculations are made for different slip lengths. The calculated volumetric flow rates and slip lengths are presented in Table 1 . The volumetric flow rate can be increased by $27 \%$ for slip length equals to channel height compared with flow rate realized for slip length of $1 \%$ height of microchannel.

Table 1. The flow rates and slip lengths.

\begin{tabular}{|c|c|}
\hline Slip length, $\mathbf{~ m m}$ & $\begin{array}{l}\text { Volumetric flow } \\
\text { rate, } \mathbf{1 0}^{\mathbf{3}} \times \mathbf{m m}^{\mathbf{3}} / \mathbf{s}\end{array}$ \\
\hline 0.005 & 188.8 \\
\hline 0.25 & 214.3 \\
\hline 0.5 & 240.5 \\
\hline
\end{tabular}




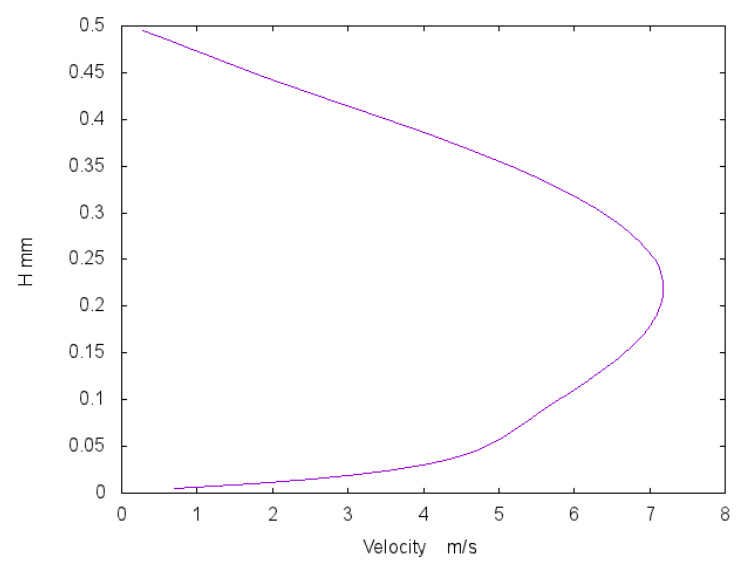

Fig. 2. The velocity profile on the no slipping surface.

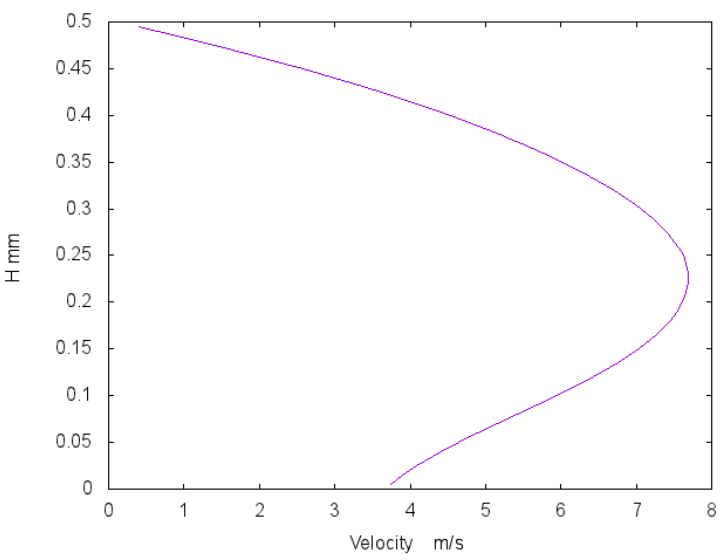

Fig. 3. The velocity profile on the hydrophobic surface.

Velocity profiles for the slip length of $0.5 \mathrm{~mm}$ are presented in Fig. 2 and Fig. 3. The maximum of a transverse component of velocity is less than a lengthwise component of velocity by three orders of magnitude. It means that the transverse component of velocity practically does not affect on the flow in microchannel with bubbles layer.

\section{Conclusions}

Results of 2D numerical solution of liquid flow in microchannel with bubbles layers on surface are presented. Bubbles layers are modeled by setting of bubble size and Navier slip condition. Calculations have been done using OpenFoam PISO method. The results of modeling compared with analytical solution.

The vertical velocity arising due to bubbles are not significant for the flow in the microchannel.

The volumetric flow rate can be increased by $27 \%$ if slip length equals to channel height compared with the flow rate realized for slip length of $1 \%$ of microchannel height.

This work was supported by a grant from the Russian Science Foundation (project Nr. 1419-01755). 


\section{References}

1. J. Ou, B. Perot, and J.P. Rothstein, Phys. Fluids. 16, 4635 (2004)

2. J.P. Rothstein, Annu. Rev. Fluid Mech. 42, 89 (2010)

3. E. Karatay, A. S. Haase, C. W. Visser, C. Sun, D. Lohse, P. A. Tsai, and R. G. H. Lammertnik, Proc. National Acad. Sci. USA 110, 8422 (2013)

4. V.S. Ajaev, E.Y. Gatapova, O.A. Kabov, Phys. Fluids 25, 122102 (2013)

5. V.S. Ajaev, E.Y. Gatapova, O.A. Kabov, Microgravity Sci. Techn. 24, 33 (2012)

6. V.S. Ajaev, E.Y. Gatapova, O.A. Kabov, Adv. Colloid interface Sci. 228, 92 (2016)

7. E.Y. Gatapova, V.S. Ajaev, O.A. Kabov, JETP Lett. 101, 160 (2015)

8. A.V. Belyaev, O.I. Vinogradova, J. Fluid Mech. 652, 489 (2010) 